\title{
A Facile Method to Enhance Photovoltaic Performance of Benzodithiophene-Isoindigo Polymers by Inserting Bithiophene Spacer
}

\author{
Zaifei Ma, Dongfeng Dang, Zheng Tang, Desta Gedefaw, Jonas Bergqvist, \\ Weiguo Zhu, Wendimagegn Mammo, Mats R. Andersson, Olle Inganäs, \\ Fengling Zhang, and Ergang Wang*
}

Polymer solar cells (PSCs) have attracted the interest of academia and industry in recent years due to their advantages of low cost fabrication by solution processing. ${ }^{[1]}$ However, there are alternatives for the design of PSCs: the bulk heterojunction (BHJ) system, ${ }^{[2]}$ which uses a blend of conjugated polymers (as the electron donor) and fullerenes (as the electron acceptor), is the most widely studied. ${ }^{[1]}$ An ideal conjugated polymer for PSCs needs to possess broad and strong absorption in the visible and near-infrared regions, high hole mobility, suitable highest occupied molecular orbital (HOMO)-lowest unoccupied molecular orbital (LUMO) energy levels and appropriate miscibility with the fullerenes. ${ }^{[1 \mathrm{a}]}$ With the design and synthesis of a large amount of polymers and intensive device optimization work, the power conversion efficiency (PCE) of PSCs has reached $7-9 \%$ over the past few years. ${ }^{[3]}$ A thorough understanding of the relationship between the chemical structures of polymers and their photovoltaic performance is necessary to significantly improve PCEs of PSCs. ${ }^{[3 a]}$ One versatile approach to construct low bandgap polymers is the combination of donor (electron rich) and acceptor (electron deficient) units, forming internal charge transfer structure. ${ }^{[1,4]}$ Benzo[1,2-b:4,5-b']dithiophene (BDT) is an electron rich unit with a rigid and planar structural feature that promotes $\pi-\pi$ stacking of the resulting

Z. Ma, Z. Tang, J. Bergqvist, Prof. O. Inganäs,

Dr. F. Zhang

Biomolecular and Organic Electronics

IFM, Linköping University

SE-581 83, Linköping, Sweden

D. Dang, Dr. D. Gedefaw, Prof. M. R. Andersson,

Dr. E. Wang

Department of Chemica

and Biological Engineering/Polymer Technology

Chalmers University of Technology

SE-412 96, Göteborg, Sweden

E-mail: ergang@chalmers.se

D. Dang, Prof. W. Zhu

Key Lab of Environment-Friendly Chemistry

and Application of the Ministry of Education

College of Chemistry Xiangtan University

Xiangtan, 411105, China

Prof. W. Mammo

Department of Chemistry

Addis Ababa University

P.O. Box 1176, Addis Ababa, Ethiopia

DOI: $10.1002 /$ aenm.201301455 polymers and hence, higher mobility can be expected. ${ }^{[5]}$ Quite many high-performance polymers are based on BDT, which makes it one of the best donor units at present. ${ }^{[5]}$ Isoindigo, a strong electron deficient unit due to its two lactam rings, has been known in the dye industry for a long time. ${ }^{[6]}$ Recently, the isoindigo unit has been successfully introduced in the field of organic electronics for constructing both donor-acceptor (D-A) small molecules ${ }^{[7]}$ and polymers. ${ }^{[8]}$ An isoindigo-based polymer, P3TI, achieved efficiency up to $6.3 \%,{ }^{[9]}$ which makes this dye a promising acceptor unit for $\mathrm{D}-\mathrm{A}$ polymers. Therefore, BDTisoindigo polymers turn out to be very interesting systems for investigating polymer structure-property relationships. It has been recognized that the insertion of one thiophene as a spacer group between D-A units can enhance performance of the resulting polymers. ${ }^{[1 \mathrm{a}, 10]}$ However, there are only few reports on the insertion of bithiophene as a spacer group. ${ }^{[11]}$ The use of terthiophene as spacer has not been reported. Thus, we conducted a systematic study on solar cell performance of BDT-isoindigo polymers as a function of the number of thiophene spacer units. Four polymers, namely, PBDT-I, PBDTTIT, ${ }^{[10 b]}$ PBDT-BTI, and PBDT-TTI (Scheme 1), based on an alkoxy-substituted BDT (donor) and alkyl-substituted isoindigo (acceptor) units, were synthesized and characterized. In PBDTI, no thiophene spacer was incorporated, while one, two and three thiophene spacers were introduced in PBDT-TIT, PBDTBTI, and PBDT-TTI, respectively. The polymers were used to fabricate solar cell devices and it was found that the polymer that contained bithiophene spacers (PBDT-BTI) showed superior performance reaching PCE up to $7.3 \%$ with [6,6]-phenyl$\mathrm{C}_{71}$-butyric acid methyl ester $\left(\mathrm{PC}_{71} \mathrm{BM}\right)$ as electron acceptor in a conventional $\mathrm{BHJ}$ device configuration. PBDT-TTI and PBDT-I showed PCEs of $3.98 \%$, and $2.80 \%$ together with $\mathrm{PC}_{71} \mathrm{BM}$ while PBDT-TIT exhibited a PCE of $4.85 \%$ together with $\mathrm{PC}_{61} \mathrm{BM}$. This result shows that insertion of bithiophene as a spacer is indeed a viable strategy to achieve high performance. Because of the fact that there is no appreciable synthetic challenge in introducing the bithiophene and terthiophene spacer units, this strategy will provide a facile access to high performing materials. We also believe that the method could be implemented to other polymer systems.

Scheme 1 shows the structures of the four polymers investigated. The syntheses of PBDT-I and PBDT-TIT were reported previously. ${ }^{[10 \mathrm{~b}]}$ The synthetic details of PBDT-BTI and PBDT-TTI are described in the Supporting Information. The thiophene 


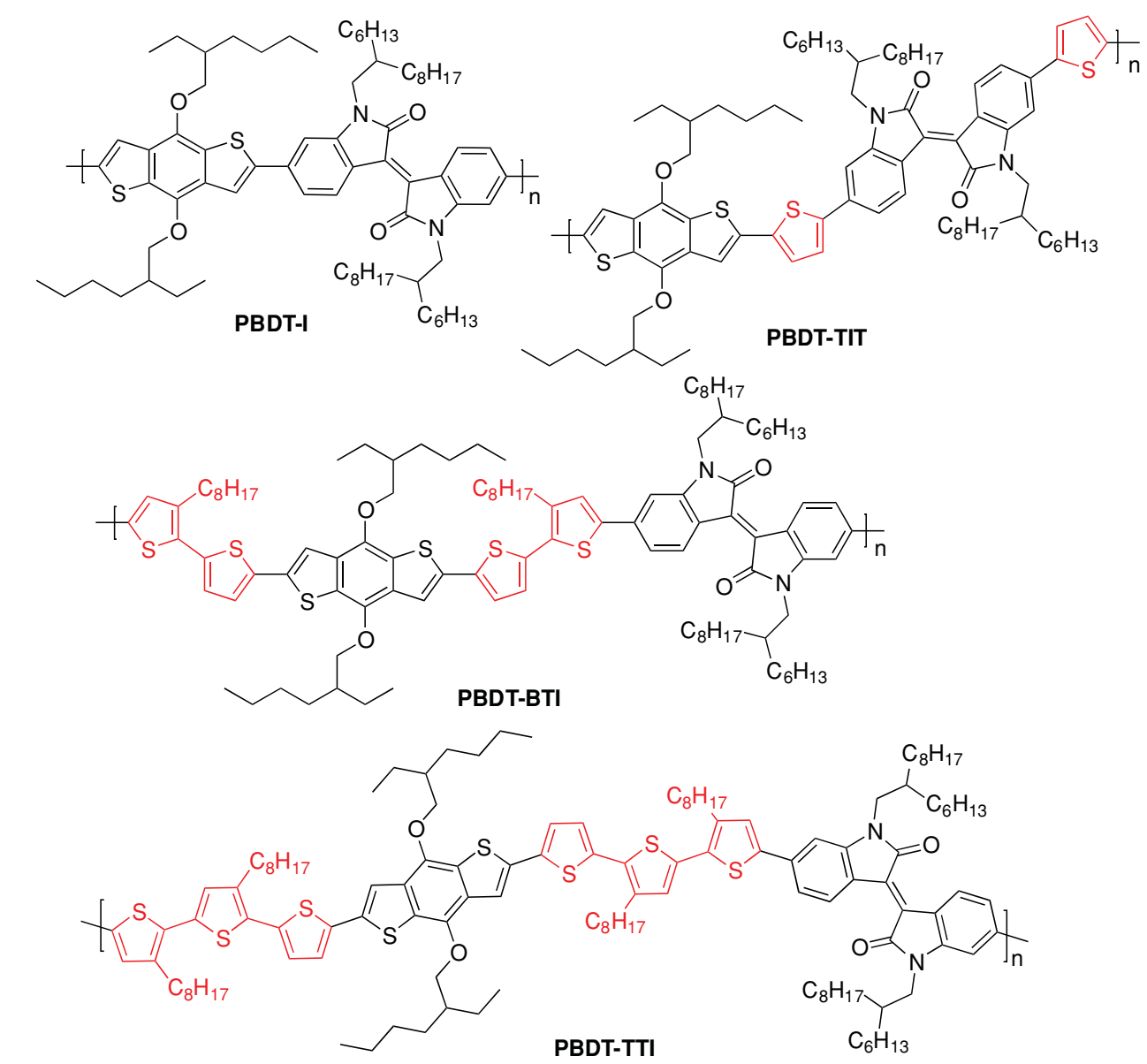

Scheme 1. Structures of PBDT-I, PBDT-TIT, PBDT-BTI, and PBDT-TTI.

spacer units were combined with the isoindigo units by Stille coupling, followed by bromination to yield the desired monomers in high yield. These monomers were polymerized with bis-stannylated BDT units via Stille coupling to afford the corresponding polymers. To ensure good solubility of PBDT-BTI and PBDT-TTI, octyl groups were attached at suitable position of thiophene rings without causing obvious steric hindrance. All the four polymers were readily soluble in organic solvents such as chloroform and o-dichlorobenzene (DCB). It was found that the number-average molecular weights $\left(M_{n}\right)$ increased with increasing number of thiophene spacer units. Thus,
PBDT-I, PBDT-TIT, PBDT-BTI, and PBDT-

TTI showed $M_{\mathrm{n}}$ of $33000,42000,55000$, and 139000 with polydispersity indices of $5.0,2.6,3.3$, and 4.5 , respectively.

Figure 1 shows the optical absorption spectra of PBDT-I, PBDT-TIT, PBDT-BTI, and PBDT-TTI in solution (a) and in the solid state (b). The absorption onsets of the four polymers measured in chloroform solution extended up to $750 \mathrm{~nm}$ with strong absorption peaks appearing at around $450 \mathrm{~nm}$ and $650 \mathrm{~nm}$. The highenergy band that appeared at $\approx 450 \mathrm{~nm}$ is attributable to a localized $\pi-\pi^{*}$ transition while the absorption band at $\approx 650 \mathrm{~nm}$ is due to intramolecular D-A charge transfer. The relative intensity of the highenergy band increased along with the conjugation length of the donor segment as shown in Figure 1a. PBDT-BTI presented red-shifted spectra compared to PBDT-TIT due to the extended donor segments. Different from PBDT-TIT, which retained the same absorption onset in going from solution to the solid state, PBDT-BTI and PBDT-TTI showed red-shifted spectra in the solid state probably due to better $\pi-\pi$ stacking of the polymer chains in the solid state.

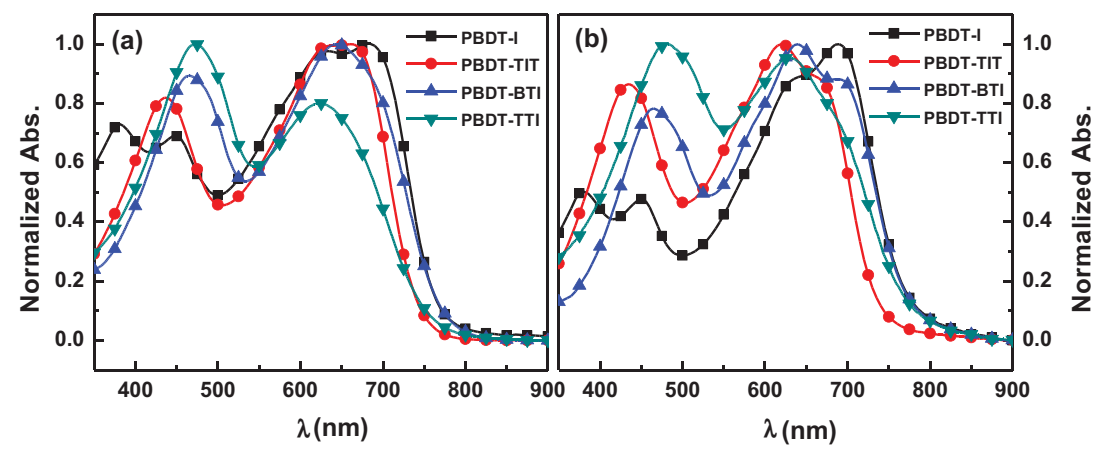

Figure 1. Absorption spectra of the polymers a) in chloroform solution and b) in the solid state. 


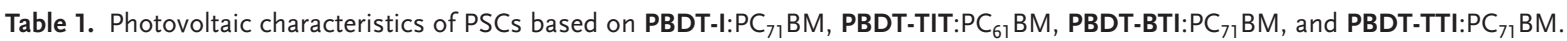

\begin{tabular}{|c|c|c|c|c|c|c|c|c|}
\hline Solvent & Active layer & $\begin{array}{c}d \\
{[\mathrm{~nm}]}\end{array}$ & $\begin{array}{c}J_{\mathrm{sc}} \\
{\left[\mathrm{mA} \mathrm{cm}^{-2}\right]}\end{array}$ & $\mathrm{FF}$ & $\begin{array}{l}V_{o c} \\
{[V]}\end{array}$ & $\begin{array}{l}\text { PCE } \\
{[\%]}\end{array}$ & $\begin{array}{c}\text { Theoretical } J_{\mathrm{sc}} \\
{\left[\mathrm{mA} \mathrm{cm}^{-2}\right]}\end{array}$ & $\begin{array}{l}\text { IQE } \\
{[\%]}\end{array}$ \\
\hline $\mathrm{DCB}+\mathrm{DIO}$ & PBDT-I:PC ${ }_{71} B M$ & 90 & 6.40 & 0.52 & 0.85 & 2.80 & 15.20 & $42 \%$ \\
\hline $\mathrm{DCB}+\mathrm{DIO}$ & PBDT-TIT:PC ${ }_{61} \mathrm{BM}$ & 95 & 9.38 & 0.66 & 0.79 & 4.85 & 14.10 & $67 \%$ \\
\hline TCB & PBDT-BTI:PC ${ }_{71} B M$ & 105 & 14.96 & 0.68 & 0.72 & 7.31 & 17.18 & $87 \%$ \\
\hline $\mathrm{DCB}+\mathrm{DIO}$ & PBDT-TTI:PC $\mathrm{C}_{71} \mathrm{BM}$ & 110 & 11.82 & 0.52 & 0.66 & 3.98 & 15.68 & $75 \%$ \\
\hline
\end{tabular}

Cyclic voltammetry (CV) was used to investigate the molecular energy levels (HOMO and LUMO) of the four polymers. The cyclic voltammograms are shown in the Supporting Information. The HOMO energy levels of PBDT-I, PBDT-TIT, PBDTBTI, and PBDT-TTI were estimated to be $-5.81,-5.75,-5.62$, and $-5.62 \mathrm{eV}$, respectively, from the onset of oxidation in the CV curves (Supporting Information Figure S1), showing that the polymers have deep HOMO levels, which is important to achieve high open-circuit voltage $\left(V_{\text {oc }}\right)$. The LUMO energy levels of the polymers are very close $(-3.95,-3.91,-3.95$, and $-3.90 \mathrm{eV}$ for PBDT-I, PBDT-TIT, PBDT-BTI, and PBDT-TTI, respectively) as deduced from the onset of reduction in the CV curves. The $\mathrm{CV}$ measurements indicated that conjugation length of the donor segment has no obvious influence on LUMO levels of the polymers but has influence on HOMO levels.

The photovoltaic performances of the four polymers as donors were investigated in BHJ solar cells with the conventional device configuration of glass/indium tin oxide (ITO)/ poly(3,4-ethylenedioxythiophene):poly(styrenesulfonate) (PEDOT:PSS)/polymer:PCBM/LiF/Al under the illumination of AM $1.5 \mathrm{G}$ simulated solar light $\left(100 \mathrm{~mW} \mathrm{~cm}{ }^{-2}\right)$. A previous study indicated that PBDT-TIT gave better performance when PC $_{61}$ BM was used as acceptor. ${ }^{[10 \mathrm{~b}]}$ However, PBDT-I, PBDT-BTI, and PBDT-TTI, performed better with $\mathrm{PC}_{71} \mathrm{BM}$ as acceptor. For all four polymers, the optimal ratios of polymer:PCBM (D:A) in the active layer were found to be $1: 1.5$. The influence of the $\mathrm{D}: \mathrm{A}$ ratio on the performance of PBDT-BTI is summarized in Supporting Information Figure S2 and Table S1. The best performing device with a D:A ratio of $1: 1.5$ gave a PCE of $7.31 \%$. It is however worth noting that PCEs over $6 \%$ were recorded with D:A ratios varying from 1:1 to $1: 2$. The best processing solvent for PBDT-I, PBDT-TIT, and PBDT-TTI was DCB with the addition of $2.5 \%$ (by volume) 1,8-diiodooctane (DIO) while PBDT-BTI gave better efficiency when 1,2,4-trichlorobenzene (TCB) was used as processing solvent without any additive. As shown in Supporting Information Figure S3 and Table S2, the performances of PBDTBTI-based PSCs processed from different solvents were quite different, which can be attributed to the different morphologies of the active layers according to previous studies. ${ }^{[12]}$ Table 1 shows a summary of the device parameters of the solar cells fabricated from the four polymers and the corresponding current density-voltage $(J-V)$ curves of the devices are shown in Figure 2a. To verify the performance reproducibility of the best-performing polymer, PBDT-BTI, 20 devices were fabricated, which gave low variation in PCE with an average PCE of $7.0 \%$ and the highest of $7.31 \%$. The related statistical boxes of the device parameters are summarized in Supporting Information Figure S4. The fact that TCB, without any additive, was used for processing the films and that no thermal or solvent annealing was required could be one of the reasons for the high reproducibility of the results. This may be an attractive feature in the processing of PBDT-BTI to prepare large area devices. It was noted that as the number of thiophene spacers increased the $V_{\text {oc }}$ of the polymers decreased from 0.85 to $0.79,0.72$, and 0.66 V for PBDT-I-, PBDT-TIT-, PBDT-BTI-, and PBDT-TTIbased solar cells, respectively. We noticed that the $V_{\text {oc }}$ of the solar cells based on PBDT-BTI- and PBDT-TTI are quite different, although the energy of the HOMO level is the same for these two polymers. This suggests that recombination rate constants for the two different systems are different. Higher recombination rate is expected in the solar cell based on PBDT-TTI, ${ }^{[13]}$ which could be related to the chemical structure of PBDT-TTI or the worse morphology of the active layer. It can be seen that the short-circuit current $\left(U_{\mathrm{sc}}\right)$ of the device prepared from PBDTBTI: $\mathrm{PC}_{71} \mathrm{BM}$ in 1:1.5 ratio reached $14.96 \mathrm{~mA} \mathrm{~cm}{ }^{-2}$, which is significantly higher than the $J_{\mathrm{sc}}$ generated from devices prepared from PBDT-TIT:PC ${ }_{61} \mathrm{BM}$ (9.38 $\left.\mathrm{mA} \mathrm{cm}{ }^{-2}\right)$, PBDT-I:PC ${ }_{71} \mathrm{BM}$ $\left(6.40 \mathrm{~mA} \mathrm{~cm}^{-2}\right)$, and PBDT-TTI:PC ${ }_{71} \mathrm{BM}\left(11.82 \mathrm{~mA} \mathrm{~cm}^{-2}\right)$. One possible cause for the different $J_{\mathrm{sc}}$ values can be the different extinction coefficients of the different active layers, as plotted in Supporting Information Figure S6a. This is consistent with the calculated maximum $J_{\mathrm{sc}}$ values for the solar cells with active layer thicknesses of $\approx 100 \mathrm{~nm}$ as listed in Table 1 . The maximum $J_{\mathrm{sc}}$ values were calculated for the solar cells using a transfer matrix model (TMM) and the measured optical constants. Internal quantum efficiencies (IQEs) were assumed to
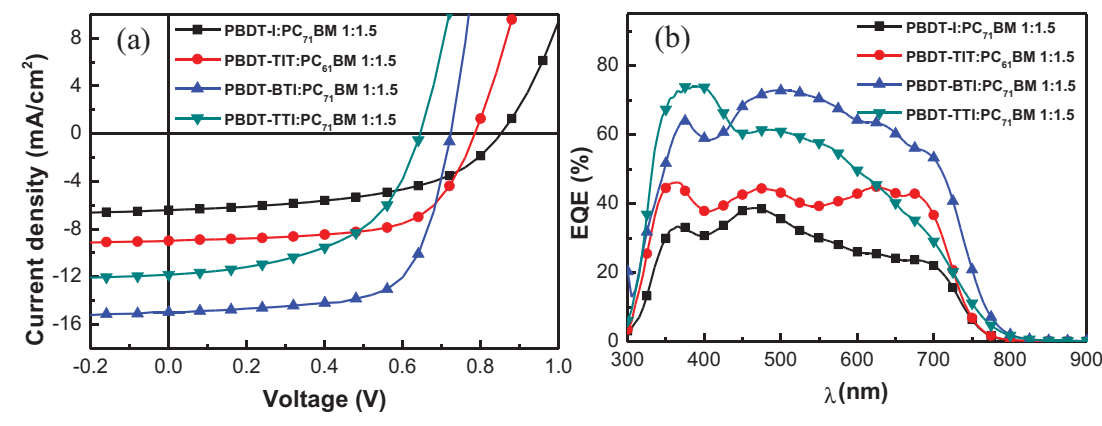

Figure 2. a) $J-V$ curves and b) EQE spectra of the PSCs based on PBDT-I:PC 71 BM, PBDTTIT:PC ${ }_{61} B M$, PBDT-BTI:PC 11 BM, and PBDT-TTI:PC ${ }_{71} B M$. 
be $100 \%$ for the solar cells in the calculation. Different morphologies of the active layers gave rise to different exciton dissociation efficiencies and thus could also be the reason for the different $J_{\text {sc }}$ values. ${ }^{[12,14]}$ This is corroborated by the fact that the average IQE values of the solar cells are significantly different in the solar cells based on different polymers. (Table 1) Here, IQE was calculated using the method described by Inganäs et $a l .{ }^{[15]}$ Considering the fact that the dependence of current generation on electric field close to short circuit conditions are very similar, the difference in IQE has to be due to the difference in exciton dissociation efficiency. Thus, morphologies of the active layers based on different isoindigo polymers are expected to be different. Atomic force microscopy (AFM) was used to investigate the surface morphologies of PBDT-I:PC ${ }_{71}$ BM, PBDTTIT:PC ${ }_{61} \mathrm{BM}$, PBDT-BTI:PC ${ }_{71} \mathrm{BM}$, and PBDT-TTI:PC ${ }_{71} \mathrm{BM}$ blends to find the influence of morphology on device performance. From Supporting Information Figure S5, it is clear that the topographies of the different active layers are different. However, it is difficult to obtain detailed information related to morphology of the active layer, as AFM measures only the surface of the active layer. Photoluminescence (PL) of photoactive polymers can be efficiently quenched when intimately blended with PCBM due to photoinduced charge transfer. ${ }^{[2]}$ A blend with smaller polymer domain size has higher PL quenching efficiency compared to that with big one. Thus, PL quenching efficiency can directly reflect polymer domain size in a blend. Here, PL spectra of the pure polymers and the corresponding polymer:PCBM blends were measured and the normalized spectra are shown in Figure 3. The PBDT-BTI:PC ${ }_{71} \mathrm{BM}$ blend shows much lower intensity relative to PBDT-BTI ( $\approx 90 \% \mathrm{PL}$ quenching efficiency), which suggests that polymer excitons can efficiently diffuse to the D:A interface and dissociate into carriers. As polymer exciton diffusion length is generally in the range of $5-20 \mathrm{~nm},{ }^{[16]}$ smaller polymer domains in PBDTBTI:PC ${ }_{71} \mathrm{BM}$ blend can be inferred. In contrast, the PBDT$\mathrm{I}: \mathrm{PC}_{71} \mathrm{BM}$ blend showed the lowest quenching efficiency, suggesting non-optimal morphology for exciton dissociation and

thus low $J_{\mathrm{sc}}$. The high $J_{\mathrm{sc}}$ and fill factor $(\mathrm{FF})$ obtained from the PBDT-BTI:PC ${ }_{71} \mathrm{BM}$ device was the result of an efficient charge carrier formation, transportation and collection. ${ }^{[17]}$ The high $J_{\mathrm{sc}}$ and FF combined with a decent $V_{\text {oc }}$ obtained from the device of PBDT-BTI:PC ${ }_{71} \mathrm{BM}$ pushed the PCE of this device to $7.3 \%$, which makes this polymer system the highest performing isoindigo-based polymer reported to date.

The external quantum efficiencies (EQEs) of the solar cell devices were also measured and demonstrated that a charge carrier is generated and collected over the region between $300 \mathrm{~nm}$ and $750 \mathrm{~nm}$ for the four polymers (Figure 2b). The device made from PBDT-BTI:PC ${ }_{71} \mathrm{BM}$ was superior, with an EQE above $60 \%$ over a wide range. The current density values integrated from the EQE curves with the AM 1.5G spectrum agreed well with the corresponding $J_{\text {sc }}$ from $J-V$ measurements. Quantum chemical calculations were performed to further understand the polymer structure-property relationship. The optimized geometries of the four polymer chains, when viewed from the side, are given in Figure S9 (Supporting Information). It was found that when two or three thiophene rings were inserted as spacers (PBDT-BTI, PBDT-TTI), the geometries of the polymer chains became perfectly planar without twisting of the repeating units compared to the polymers consisting one and no thiophene as spacer. Obviously, the planar structure observed in PBDT-BTI improved intramolecular donor-acceptor charge transfer effect, as indicated by its high absorption coefficient (Supporting Information Figure S6a), and interpolymer chain interaction, which may enhance mobility of charge carriers. ${ }^{[18]}$ Although PBDT-TTI also possessed planar backbones, the incorporation of too many thiophene rings reduced the content of the electron-withdrawing isoindigo group, leading to weak intramolecular donor-acceptor charge transfer effect and thus low absorption coefficient (Supporting Information Figure S6a). It was noted that PBDT-TTI exhibited much higher molecular weight than the other three, but it is hard to expect higher performance from PBDT-TTI with lower molecular weight due to its low absorption coefficient and poor morphology in blends.

The most efficient PSCs were achieved
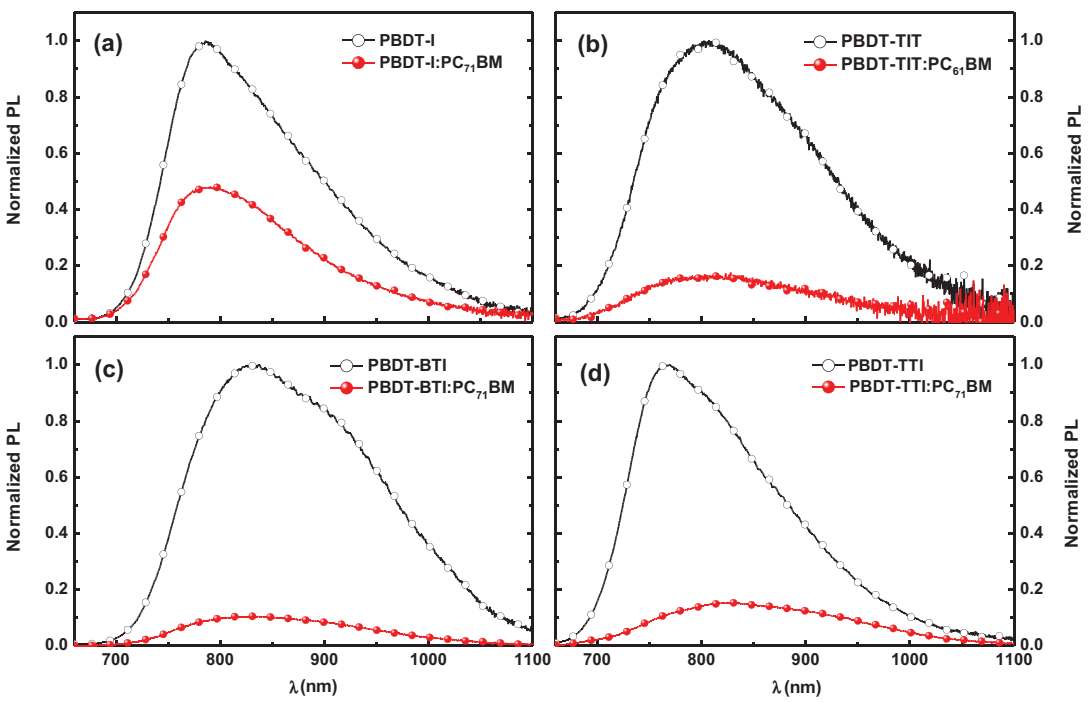

Figure 3. PL spectra obtained from pure and blend films of a) PBDT-I, b) PBDT-TIT, c) PBDTBTI, and d) PBDT-TTI systems. with very thin active layers $(80-120 \mathrm{~nm})$. However, thicker layers $(>200 \mathrm{~nm})$ are preferred for large area printing of PSCs since very thin active layer can easily have pinhole defects and suffer from low performance reproducibility. ${ }^{[19]}$ Therefore, it is important to investigate the performance of a new polymer with different thicknesses of the active layer. We used both TMM simulation $^{[20]}$ and experimental techniques to investigate the solar cell performance. The refractive index $n$ and absorption coefficient $k$ of PBDT-BTI:PC ${ }_{71}$ BM were obtained by modeling the data from ellipsometry measurements over the spectral range of absorption. They were used as computer input to simulate maximum photocurrent density $\left(J_{\text {sc-max }}\right)$, assuming all the absorbed photons are converted into charges. A plot of the simulated $J_{\text {sc-max }}$ as a function of active layer thickness 


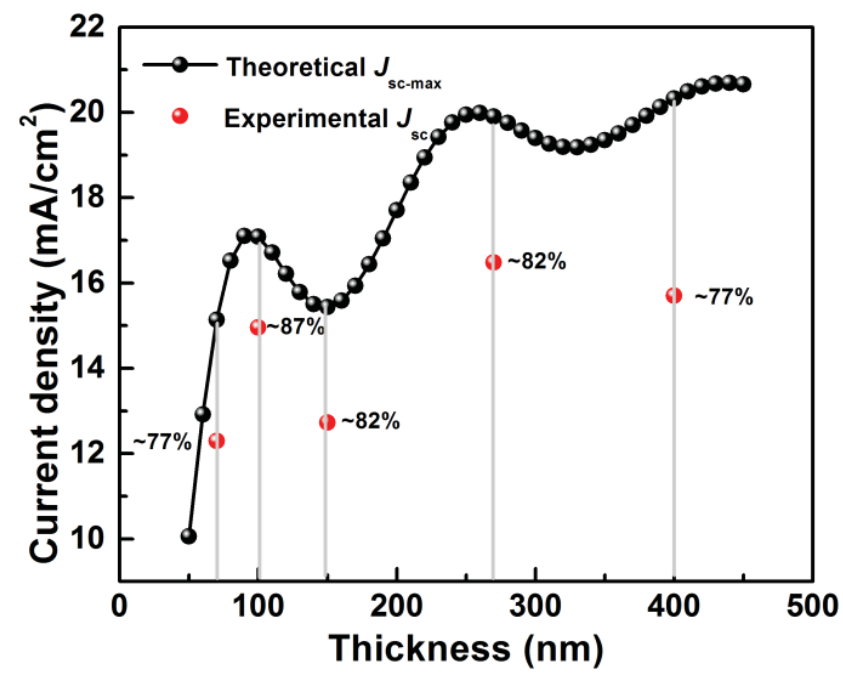

Figure 4. The theoretical $J_{\text {sc-max }}$ and experimental $J_{\mathrm{sc}}$ of PBDT-BTI:PC $\mathrm{P}_{71} \mathrm{BM}$ as a function of active layer thickness.

is shown in Figure 4. Solar cells with five key active layer thicknesses of $70,105,145,270$, and $400 \mathrm{~nm}$ were fabricated, where those with thicknesses of 105, 270, and $400 \mathrm{~nm}$ corresponded with the three interference maxima and that with $145 \mathrm{~nm}$ thickness corresponded with the first interference minimum observed from the simulation results as depicted in Figure 4. The corresponding $J-V$ and EQE curves are shown in Supporting Information Figure S7 and the parameters are summarized in Supporting Information Table S3. For comparison, the experimental $J_{\mathrm{sc}}$ have also been included in Figure 4. We found that the experimental $J_{\mathrm{sc}}$ values were in good agreement with the simulated $J_{\text {sc-max }}$ values with an average of $82 \% \pm 5 \%$. It is striking to note that an efficiency up to $6.3 \%$ could be achieved by solar cells with an active layer as thin as $70 \mathrm{~nm}$, which benefited from the high absorption coefficient $\left(1.43 \times 10^{5} \mathrm{~cm}^{-1}\right.$ at $634 \mathrm{~nm}$ ) of PBDT-BTI. On the other hand, a thickness of $270 \mathrm{~nm}$, which fits the requirement for large area printing of PSCs, could still afford a PCE over 6\%. To further understand the loss mechanism of the solar cells, IQE spectra of solar cells with different active layer thickness were calculated (the details are described in the Supporting Information). As shown in Supporting Information Figure S8, the IQEs of solar cells based on PBDT-BTI:PC ${ }_{71}$ BM do not strongly depend on the thickness of the active layer. For the solar cell with an optimal thickness of $105 \mathrm{~nm}$, an average IQE value of $\approx 90 \%$ was obtained with a maximum IQE value approaching $100 \%$ at $550 \mathrm{~nm}$, which suggests that nearly every absorbed photon dissociates into free charge carriers and the generated carriers are finally collected at the electrodes. For the solar cell based on a $400 \mathrm{~nm}$ active layer, the average IQE remained as high as $\approx 80 \%$, most likely due to the high carrier mobility of isoindigo-based polymers. ${ }^{[21]}$

In conclusion, we have described the synthesis and characterization of four polymers based on BDT and isoindigo with zero, one, two, and three thiophene spacer groups. We have demonstrated that the use of bithiophene as a spacer unit improves the geometry of the polymer chain, making it become planar, and hence potentially enhanced $\pi-\pi$ stacking occurs. As a result of the favorable interaction of the polymer chains, enhanced absorption coefficient, and optimal morphology, PBDT-BTI, which possesses bithiophene as a spacer, revealed high current and fill factor leading to a PCE of $7.3 \%$ in devices, making this polymer the best performing isoindigo-based material in PSCs. Moreover, PBDT-BTI could still maintain efficiency of over $6 \%$ with the active layer thickness of $270 \mathrm{~nm}$, which makes it a promising candidate for a material in printed PSCs. Thus, the use of thiophene spacers in D-A polymers could be an important design strategy to produce high-performance polymers for solar cells.

\section{Experimental Section}

Solar Cell Device Fabrication and Characterization: $\mathrm{BH}$ PSCs were fabricated using a conventional geometry (glass/ITO/ PEDOT:PSS(40 nm)/active layer/LiF(0.6 nm)/Al(100 nm)). The ITO glass substrates were cleaned by detergent and TL-1 (a mixture of water, ammonia (25\%), and hydrogen peroxide (28\%) (5:1:1 by volume)) in turn. PEDOT:PSS 4083 (Heraeus Precious Metals $\mathrm{GmbH}$ $\&$ Co. KG) was spin-coated on top of the cleaned ITO glass substrates. Then, the substrates were heated on the heater for $15 \mathrm{~min}$ at $120{ }^{\circ} \mathrm{C}$ to remove the remaining water. Coated with PEDOT:PSS 4083, the substrates were then transferred into a glove box filled with $\mathrm{N}_{2}$, where the active layers were spin-coated from DCB, DCB:DIO (2.5 vol\%), TCB or TCB:DIO (2.5 vol\%) solutions atop PEDOT:PSS 4083 layer. The substrates with the active layer were then moved into a vacuum chamber, which was mounted in a glove box. $0.6 \mathrm{~nm} \mathrm{LiF}$ and $100 \mathrm{~nm}$ Al were then thermally evaporated under a pressure less than $4 \times$ $10^{-6}$ mbar. The active area of the PSCs was around $0.04 \mathrm{~cm}^{2}$, which was determined by optical microscopy. The $J-V$ curves were measured using a Keithley 2400 Source Meter under AM1.5 G illumination from a solar simulator (Model SS-50A, Photo Emission Tech., Inc.) with an intensity of $100 \mathrm{~mW} \mathrm{~cm} \mathrm{~cm}^{-2}$. EQE spectra of the PSCs were recorded by using a Newport Merlin lock-in amplifier. The chopped monochromatic light was illuminated on the PSCs through the transparent side. All thicknesses of the active layers were determined using a Dektak surface profiler.

\section{Supporting Information}

Supporting Information is available from the Wiley Online Library or from the author.

\section{Acknowledgements}

Z.M. and D.D. contributed equally to this work. This work was supported by the Swedish Research Council and Swedish Energy Agency.

Received: September 24, 2013 Published online:

[1] a) Y. F. Li, Acc. Chem. Res. 2012, 45, 723; b) G. Li, R. Zhu, Y. Yang, Nat. Photonics 2012, 6, 153.

[2] G. Yu, J. Gao, J. C. Hummelen, F. Wudl, A. J. Heeger, Science 1995, 270,1789

[3] a) Z. C. He, C. M. Zhong, X. Huang, W. Y. Wong, H. B. Wu, L. W. Chen, S. J. Su, Y. Cao, Adv. Mater. 2011, 23, 4636; b) C. Cabanetos, A. El Labban, J. A. Bartelt, J. D. Douglas, W. R. Mateker, J. M. J. Fréchet, M. D. McGehee, P. M. Beaujuge, J. Am. Chem. Soc. 2013, 135, 4656. 
c) M. A. Green, K. Emery, Y. Hishikawa, W. Warta, E. D. Dunlop, Prog. Photovoltaics. 2013, 21, 1.

[4] a) L. Pandey, C. Risko, J. E. Norton, J.-L. Brédas, Macromolecules 2012, 45, 6405; b) Z.-G. Zhang, J. Wang, J. Mater. Chem. 2012, 22, 4178.

[5] a) L. J. Huo, S. Q. Zhang, X. Guo, F. Xu, Y. F. Li, J. H. Hou, Angew. Chem. Int. Ed. 2011, 50, 9697; b) L. J. Huo, J. H. Hou, Polym. Chem. 2011, 2, 2453.

[6] E. W. FitzHugh, Artists Pigments: A Handbook of Their History and Characteristics, Oxford University Press, New York 1997.

[7] J. Mei, K. R. Graham, R. Stalder, J. R. Reynolds, Org. Lett. 2010, 12, 660.

[8] a) R. Stalder, J. Mei, J. R. Reynolds, Macromolecules 2010, 43, 8348; b) E. G. Wang, Z. F. Ma, Z. Zhang, P. Henriksson, O. Inganäs, F. L. Zhang, M. R. Andersson, Chem. Commun. 2011, 47, 4908.

[9] E. G. Wang, Z. Ma, Z. Zhang, K. Vandewal, P. Henriksson, O. Inganäs, F. Zhang, M. R. Andersson, J. Am. Chem. Soc. 2011, $133,14244$.

[10] a) Y. Huang, F. Liu, X. Guo, W. Zhang, Y. Gu, J. Zhang, C. C. Han, T. P. Russell, J. Hou, Adv. Energy Mater. 2013, 3, 930; b) Z. Ma, E. G. Wang, M. E. Jarvid, P. Henriksson, O. Inganäs,F. Zhang, M. R. Andersson, J. Mater. Chem. 2012, 22, 2306; c) S. Li, B. Zhao, Z. He, S. a. Chen, J. Yu, A. Zhong, R. Tang, H. Wu, Q. Li, J. Qin, Z. Li, J. Mater. Chem. A 2013, 1, 4508; d) X. F. Xu, P. Cai, Y. Lu, N. S. Choon, J. W. Chen, X. Hu, B. S. Ong, J. Polym. Sci. Polym. Chem. 2013, 51, 424; e) S. W. Shi, P. Jiang, S. Chen, Y. P. Sun, X. C. Wang, K. Wang, S. L. Shen, X. Y. Li, Y. F. Li, H. Q. Wang, Macromolecules 2012, 45, 7806.f) J. Min, Z.-G. Zhang, S. Zhang, M. Zhang, J. Zhang, Y. Li, Macromolecules 2011, 44, 7632.
[11] a) E. G. Wang, M. Wang, L. Wang, C. H. Duan, J. Zhang, W. Z. Cai, C. He, H. B. Wu, Y. Cao, Macromolecules 2009, 42, 4410; b) M. J. Zhang, X. Guo, Y. F. Li, Macromolecules 2011, 44, 8798.

[12] a) J. K. Lee, W. L. Ma, C. J. Brabec, J. Yuen, J. S. Moon, J. Y. Kim, K. Lee, G. C. Bazan, A. J. Heeger, J. Am. Chem. Soc. 2008, 130, 3619; b) X. L. Liu, S. Huettner, Z. X. Rong, M. Sommer, R. H. Friend, Adv. Mater. 2012, 24, 669.

[13] W. Tress, K. Leo, M. Riede, Phys. Rev. B. 2012, 85, 155201

[14] J. C. Bijleveld, V. S. Gevaerts, D. Di Nuzzo, M. Turbiez, S. G. J. Mathijssen, D. M. de Leeuw, M. M. Wienk, R. A. J. Janssen, Adv. Mater. 2010, 22, E242.

[15] L. A. A. Pettersson, L. S. Roman, O. Inganas, J. Appl. Phys. 2001, 89, 5564.

[16] a) P. W. M. Blom, V. D. Mihailetchi, L. J. A. Koster, D. E. Markov, Adv. Mater. 2007, 19, 1551; b) J. J. M. Halls, K. Pichler, R. H. Friend, S. C. Moratti, A. B. Holmes, Appl. Phys. Lett. 1996, 68, 3120.

[17] H.-C. Chen, Y.-H. Chen, C.-C. Liu, Y.-C. Chien, S.-W. Chou, P.-T. Chou, Chem. Mater. 2012, 24, 4766.

[18] T. Lei, Y. Cao, X. Zhou, Y. Peng, J. Bian, J. Pei, Chem. Mater. 2012, 24, 1762.

[19] a) W. Li, K. H. Hendriks, W. S. Roelofs, Y. Kim, M. M. Wienk, R. A. Janssen, Adv. Mater. 2013, 25, 3182; b) R. Sondergaard, M. Hosel, D. Angmo, T. T. Larsen-Olsen, F. C. Krebs, Mater. Today 2012, 15, 36.

[20] L. A. A. Pettersson, L. S. Roman, O. Inganäs, J. Appl. Phys. 1999, 86, 487.

[21] a) T. Lei, Y. Cao, Y. Fan, C.-J. Liu, S.-C. Yuan, J. Pei, J. Am. Chem. Soc. 2011, 133, 6099; b) J. Mei, H. Kim do, A. L. Ayzner, M. F. Toney, Z. Bao, J. Am. Chem. Soc. 2011, 133, 20130. 